\title{
Long period oscillations in the inter-plume regions of the Sun
}

\author{
D. Banerjee ${ }^{1}$, E. O’Shea ${ }^{2}$, J. G. Doyle ${ }^{3}$, and M. Goossens ${ }^{1}$ \\ 1 Centre for Plasma Astrophysics, Katholieke Universiteit Leuven, Celestijnenlaan 200B, 3001 Heverlee, Belgium \\ 2 ESA Space Science Dept., ESTEC Solar System Div., Keplerlaan 1, 2201 AZ Noordwijk, The Netherlands \\ 3 Armagh Observatory, College Hill, Armagh BT61 9DG, N. Ireland \\ e-mail: eoshea@so.estec.esa.nl; jgd@star.arm.ac.uk
}

Received 7 March 2001 / Accepted 14 August 2001

\begin{abstract}
We examine long spectral time series of inter-plume lanes observed on the 14th and 15th March 2000 with the Coronal Diagnostic Spectrometer (CDS) on-board SoHO. The observations were obtained in lines over a wide temperature range, from the chromosphere to the corona. The statistical significance of the oscillations was estimated by using a randomisation method. Our observations indicate the presence of compressional waves with periods of 20-50 min or longer, both off-limb and on-disk and up to 70 min further out to at least 25 arcsec off-limb. To our knowledge this is the first time that long period oscillations in the inter-plume regions close to the limb of the Sun have been detected. We interpret these oscillations as outward propagating slow magneto-acoustic waves which may contribute significantly to the heating of the lower corona by compressive dissipation and which may also provide some of the enough energy flux required for the acceleration of the fast solar wind. These slow waves may have been produced at the network boundaries in the coronal hole.
\end{abstract}

Key words. Sun: corona - Sun: oscillations - Sun: UV radiation

\section{Introduction}

When viewed off-limb, polar plumes are the most prominent structures in polar coronal holes (DeForest et al. 1997; Koutchmy \& Bocchialini 1998). More recently, DeForest et al. (2001) produced images from LASCO/SoHO, which clearly show polar plumes extending to altitudes of 30 solar radii or more, very close to the outer edge of the C-3 field of view and above the likely Alfvenic point of the wind flow. Plumes are considered to be bright threads rooted in the network and are thought to form at the network boundaries where small bipoles within a coronal hole reconnect with unipolar flux concentrations (Wang \& Sheely 1995; Wang 1998). It has been known that the density of the plumes are much higher (3-5 times) than the inter-plume background (Saito 1965; Ahmed \& Withbroe 1977; Young et al. 1999) implying that the coronal heating rate is not uniform over the coronal hole.

Plume formation involves magnetic reconnection between unipolar flux concentrations and nearby bipoles, but the bipolar fields constitute only a small fraction of the total magnetic flux within the polar coronal hole. Primarily,

Send offprint requests to: D. Banerjee,

e-mail: dipu@wis.kuleuven.ac.be coronal holes are overwhelmingly unipolar. This fact further suggests that plumes are not the source of the high speed polar wind (Wang et al. 1997).

The large outflow velocity as measured by Giordano et al. (2000) and Patsourakos \& Vial (2000) also favours inter-plume lanes as being the main source of the fast solar wind. The excess broadening of spectral line profiles in the inter-plumes as compared to the plumes (Hassler et al. 1997; Wilhelm et al. 1998; Banerjee et al. 2000a) further supports this idea. These authors also reported an anticorrelation between the intensity and line widths of the O VI $1032 \AA$ line in both plume and inter-plume regions, with detailed plume structures being seen out to $1.5 R_{\odot}$. Thus it is becoming increasingly important to study the dynamics of the inter-plume regions in polar coronal holes.

There are several reports on the structure, formation, evolution and dynamics of plumes in the literature, but there are only a few studies on inter-plume regions, mainly because of the fact that they are very difficult to observe spectroscopically. Banerjee et al. (2000b,c) investigated the temporal behaviour of polar plumes as observed in the transition region line, O v $629 \AA$. In this paper we report on the temporal behaviour of the inter-plume lanes as observed by the CDS/SoHO instrument. Observations were performed for lines over a wide temperature range, from 


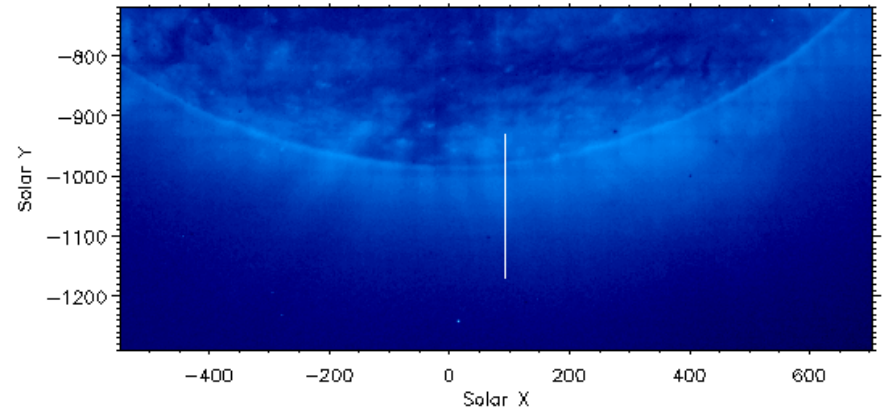

Fig. 1. Position of the observing slit for the s18831r00 dataset (14 March 2000) on an EIT/SoHO image of the south polar coronal hole in Fe IX/x $171 \AA$ taken 3 hours after the temporal series (courtesy of the EIT consortium).

the low chromosphere (He I $584 \AA$ ), through the transition region (O III $599 \AA$, O IV $554 \AA$, O v $629 \AA$ ) to the corona ( Mg IX $368 \AA$ ), in a south polar coronal hole region.

\section{Observations and data reduction}

The Coronal Diagnostic Spectrometer (CDS) on-board the Solar Heliospheric Observatory (SoHO) is a dual extreme ultraviolet spectrometer, covering the wavelength range 150 to $780 \AA$, comprising of a normal incidence and a grazing incidence spectrometer (Harrison et al. 1995). The normal incidence spectrometer (NIS), whose data is the subject of this paper, gives spectral images in two wave-bands (308-381 $\AA$ and 513-633 $\AA$ ). In order to get good time resolution, we used the NIS in a sit-andstare mode. As the slit location, $x=92, y=-1051$, is the location of the centre of the slit (for the s18831r00 dataset), the coordinates at the top of the slit, $x=92$, $y=-931$ are on the disk and thus we can work out the solar rotation expected at these coordinates. We find that by using the routine $\mathrm{ROT}_{-} \mathrm{XY}$ in the SoHO software tree that over one hour the Sun should rotate by 0.82 arcsec per hour. Therefore over 4 hours at this location we can thus expect that the Sun will rotate by a maximum of $\sim 3$ arcsec through the slit. If the source being observed has a width of 3 arcsec or greater the effect of the solar rotation on the resulting power spectrum will not be important. Furthermore, we note that the rotation velocity of 0.82 arcsec per hour is the rotation velocity at the top of the slit, i.e. this is the maximum rotation velocity. Therefore at other locations further out along the slit we would expect the effect of the sit and stare mode, on the resulting power, to be reduced further, and to become less important in the areas beyond the limb. So for our inter-plume observations in the polar region the effects of the solar rotation are not considered to be important. For the data reported here, the $2 \times 240$ arcsec slit was used. Figure 1 shows an image of the Southern polar coronal hole region taken with EIT/SoHO in Fe IX/X $171 \AA$ at 19:00 UT on March 14, 2000, with the slit superimposed (for dataset s18831r00). This figure confirms that our observations were pointed at an inter-plume region between two plume regions. One should also note that the slit is only a few arcsec away from a plume structure and intersects the plume $\sim 40$ arcsec off-limb. The consequences of this fact will be discussed in the final section.

The data discussed here were selected from 14-15 March 2000. The temporal series datasets were obtained for the five lines of He I $584 \AA(\log T=4.3 \mathrm{~K})$, O III $599 \AA,(\log T=5.0 \mathrm{~K})$, O IV $554 \AA(\log T=5.2 \mathrm{~K})$, $\mathrm{O} \vee \mathrm{V} 629 \AA(\log T=5.4 \mathrm{~K})$ and $\mathrm{Mg}$ IX $368 \AA$ $(\log T=6.0 \mathrm{~K})$, all with exposure times of $60 \mathrm{~s}$ (see Table 1 for details). Data were obtained after the recovery of SoHO and so the lines show the characteristic broadened wings. This broadening has the effect of blending the Mg IX $368 \AA$ line with that of the nearby Mg VII $367 \AA$ line. We were able to fit the Mg IX component of this blend satisfactorily by using a double Gaussian, and by fixing the position of the $\mathrm{Mg}$ VII line. We fitted the O IV $554 \AA$ line with three Gaussians to take account of the two weaker components of the $\mathrm{O}$ IV multiplet at $\sim 553$ and $555 \AA$ A. In all other cases, fitting was done using a single Gaussian as the lines were found to be generally symmetric. Details on the CDS reduction procedure, plus the Wavelet analysis, may be found in O'Shea et al. (2001) and Banerjee et al. (2001). The statistical significance of the observed oscillations was estimated by using a Monte Carlo or randomisation method. The advantage of using a randomisation test is that it is distribution free or nonparametric, i.e. it is not limited or constrained by any specific noise models, such as Poisson, Gaussian etc. We follow the method of Fisher randomisation as outlined in Nemec \& Nemec (1985) and implemented in the UK Starlink software package, PERIOD (Dhillon \& Privett 1997) (details can be found in O'Shea et al. 2001). Briefly, the randomisation test is based on the assumption that, if there is no periodic signal in the time series data, then the measured values (intensity, velocity, etc.) are independent of their observation times. For example, the intensities $I_{1}$, $I_{2}, \ldots I_{n}$, observed at times $t_{1}, t_{2}, \ldots t_{n}$, are just as likely to have occurred in any other order $I_{r(1)}, I_{r(2)}, \ldots I_{r(n)}$, where $n$ is the total number of observations and $r(1)$, $r(2), \ldots r(n)$ is a random permutation of the subscripts 1 , $2, \ldots n$. By using the maximum power peak in the global wavelet spectrum, which is just the average of the wavelet power over time and similar to a smoothed Fourier power spectrum (Torrence \& Compo 1998), at the "test statistic" (see Dhillon \& Privett 1997) it was possible to test the hypothesis that there was no periodicity in our data. The proportion of permutations that gave a value greater or equal to the peak power of the original time series would then provide an estimate of $p$, the probability that no periodic component is present in the data, i.e. a large value of $p$ suggests that there is little or no real periodicity in the data whereas a small value of $p$ suggests that the measured periodicity is likely to be real. In practise $n$ ! is usually so large that it is not possible to do this, due to computational and time constraints, and so the peak powers are generally calculated for only a random sample of $m$ permutations. By carrying out this 


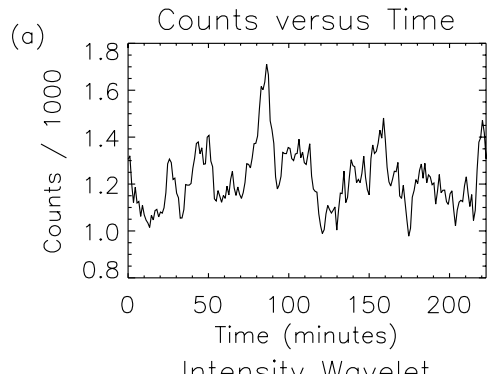

Global freq. at max. power $(>0.21 \mathrm{mHz})$

Prob. level: $99-100 \%$

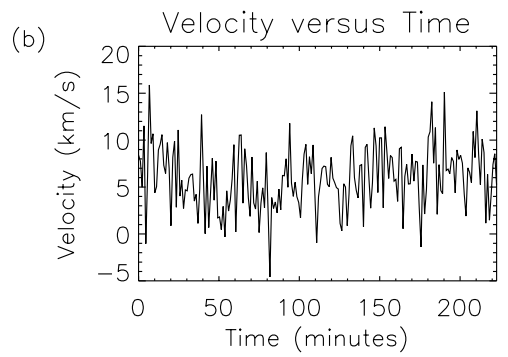

Global freq. at max.

power $(>0.21 \mathrm{mHz})$
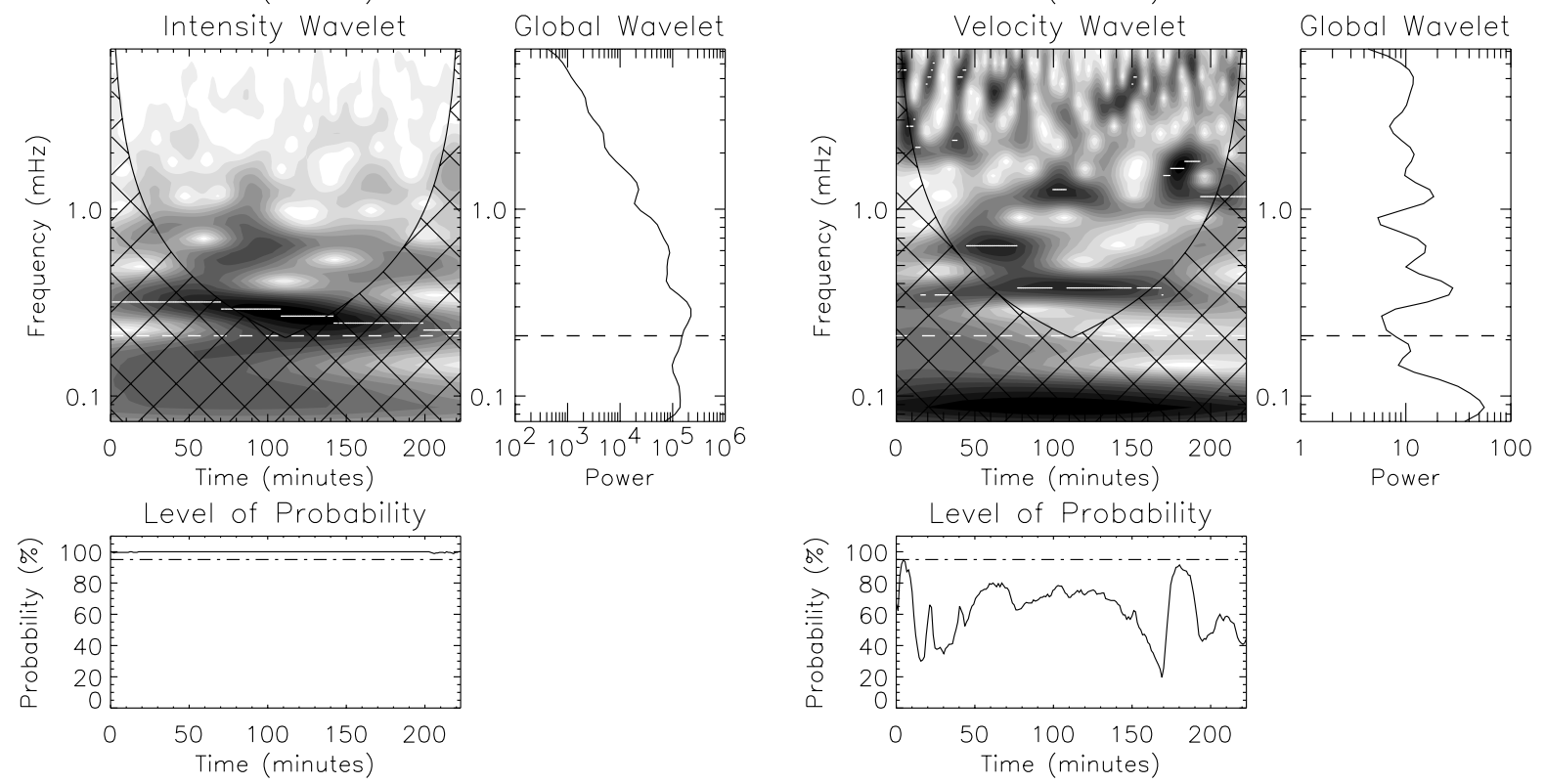

Fig. 2. Wavelet results for an inter-plume location corresponding to the He I $584 \AA$ line in the s18831r00 dataset at pixel 40 . Panels a) and b) represent intensity and velocity results respectively. The middle left panels show the time frequency phase plot corresponding to the variations shown in the top panels. The white horizontal lines in the phase plot indicate the power maxima, corresponding to the maximum probability level for that particular time. The middle right hand panels show the average of the wavelet power spectrum over time, i.e. the global wavelet spectrum. The continuous dashed horizontal lines in the wavelet spectra indicate the lower cut off frequency. The lowest panels show the variation of the probability with time from the randomisation test.

approximation, for a random sample of 250 permutations, we were able to obtain a reliable estimate of $p$. For a sample of 250 random permutations the standard errors of the $p$ values are no greater than 0.04 (Nemec \& Nemec 1985). Of course, the larger the number of permutations chosen, $m$, the lower the standard error of the $p$ value. The probability levels displayed in this paper are the values of $(1-p) \times 100$. We choose a value of $95 \%$ as the lowest acceptable probability level. Occasionally the estimated $p$ value can have a value of zero, i.e. there being an almost zero chance that the observed time series oscillations could have occurred by chance. In this case, and following Nemec \& Nemec (1985), the 95\% confidence interval can be obtained using the binomial distribution, and is given by $0.0<p<0.01$, that is, the probability $((1-p) \times 100)$ in this case is between $99-100 \%$.

To improve the signal-to-noise ratio of this data we binned by three pixels along the slit (i.e. $3 \times 1.68$ arcsec), in effect creating new pixels of $\sim 5 \times 2 \operatorname{arcsec}^{2}$. To determine the Doppler shifts, wavelength calibration is needed. We use the "limb method", where we assume that above the limb all (non-radial) wave or mass motions on average cancel out. In the absence of radiative transfer effects, the off-limb Doppler shift must on average be zero.

\section{Results}

The pointing of the slit was positioned at the south polar limb (see Fig. 1) in such a way that a major part of the slit was outside the limb and a part on the coronal hole. We first present results from the inter-plume regions (off-limb) and later on focus on the disk part of the coronal hole. We choose a $5 \times 2 \operatorname{arcsec}^{2}$ region (a single binned pixel) in an inter-plume location about 5 arcsec off limb (pixel 40, in our binned scale) for dataset s18831r00. Other regions will be discussed later.

For the He I $584 \AA$ line, formed in the low chromosphere we present the wavelet results in Fig. 2. The intensity and velocity variations are shown in the top panels. In the wavelet spectrum, the dark contour regions show the locations of the highest power. The light white horizontal lines within the dark contour regions indicate the 


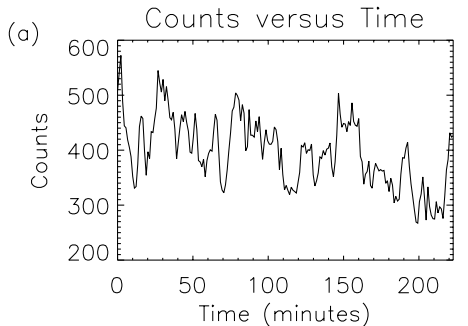

Global freq. at max. power $(>0.21$
$=0.25 \mathrm{mHz}$ Prob. level: $99-100 \%$
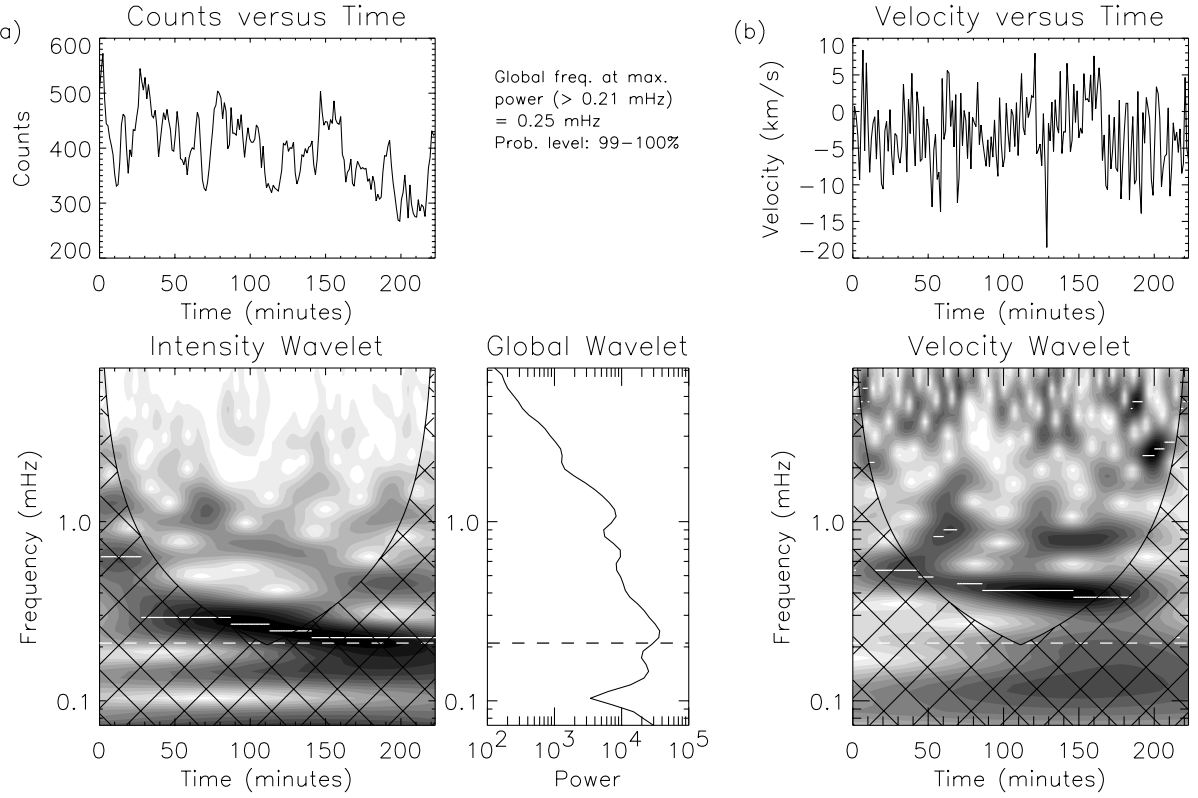
Global freq. at max
power $(>0.21 \mathrm{mHz})$

Prob. level: $98.0 \%$
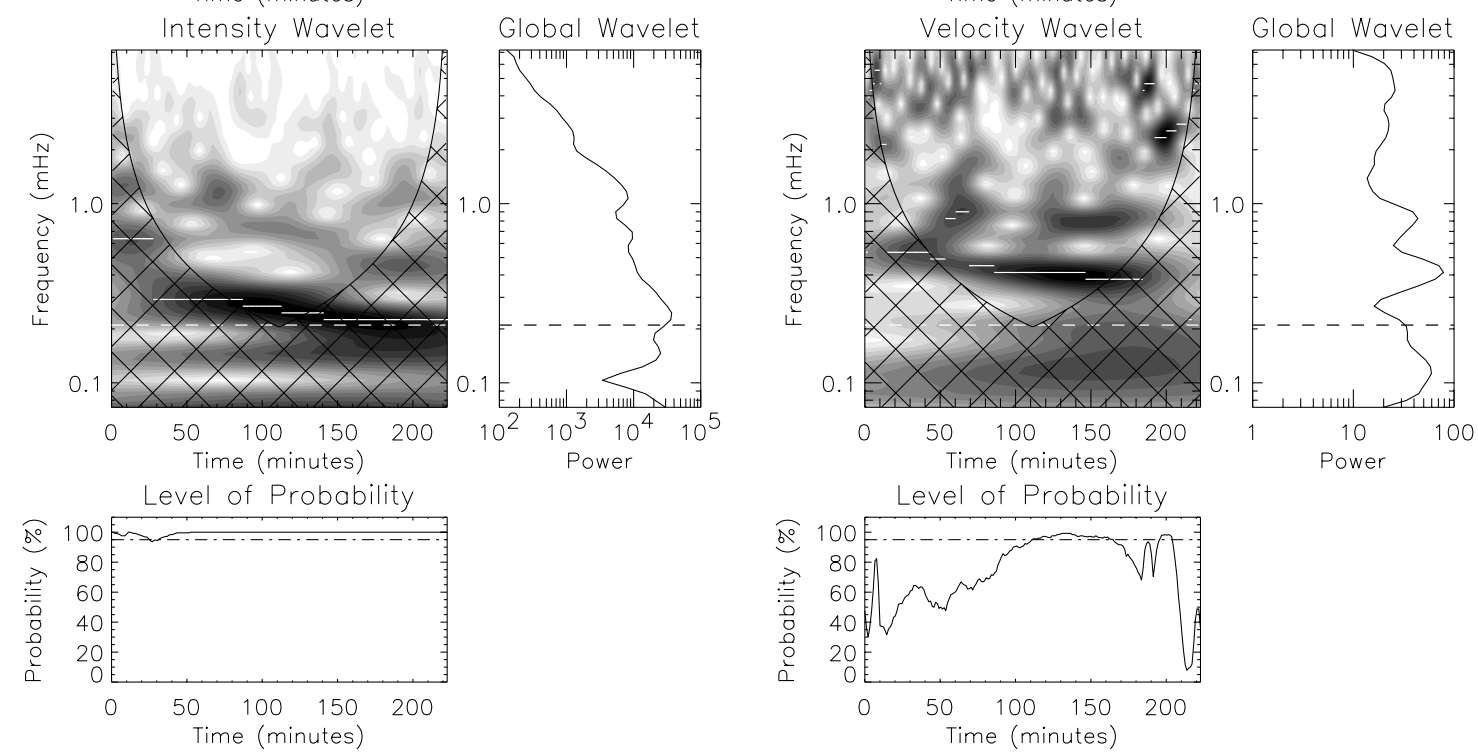

Fig. 3. The results of the wavelet analysis corresponding the O III $599 \AA$ line at pixel 40 in s18831r00. Representations are the same as Fig. 2.

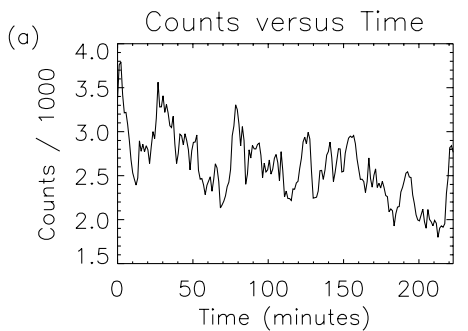

Global freq. ot max. power (>0.21 mHz) $=0.25 \mathrm{mHz}$ Prob. level: $99-100 \%$
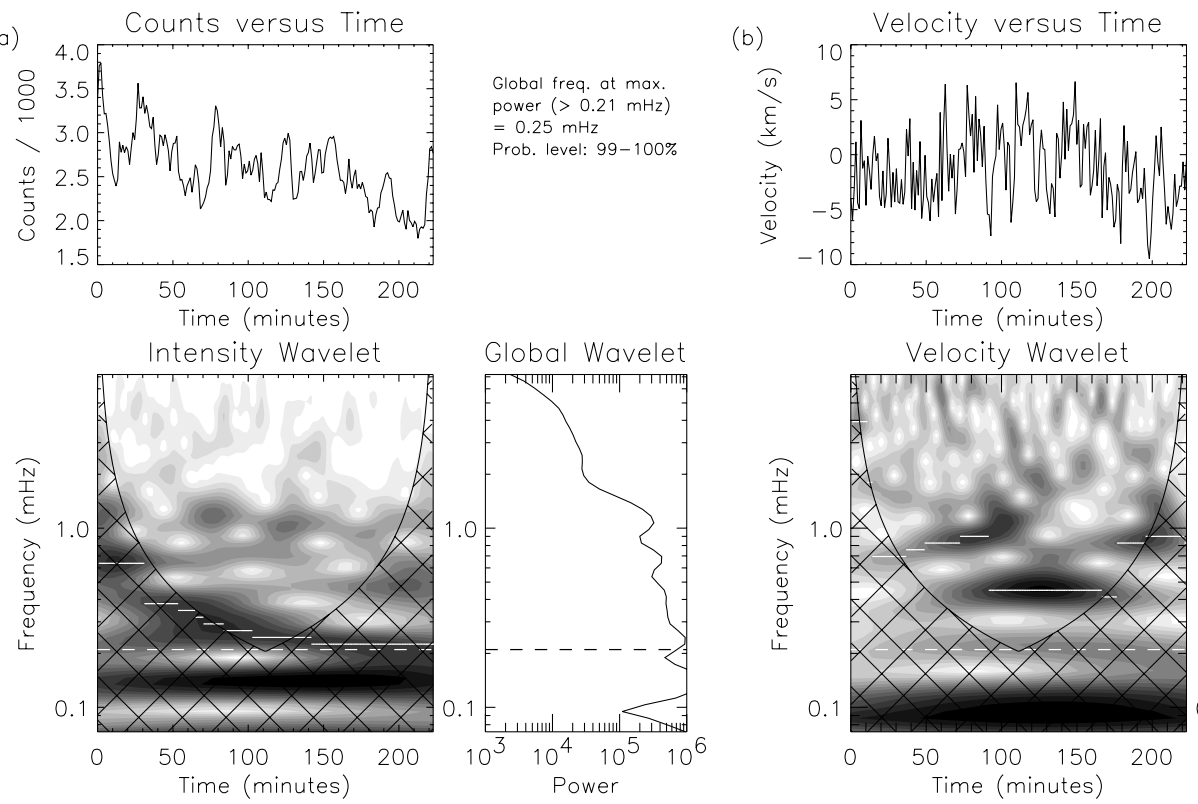

Global freq. at max.

power $(>0.21 \mathrm{~m}$
$=0.45 \mathrm{mHz}$

Prob. level: $99.6 \%$
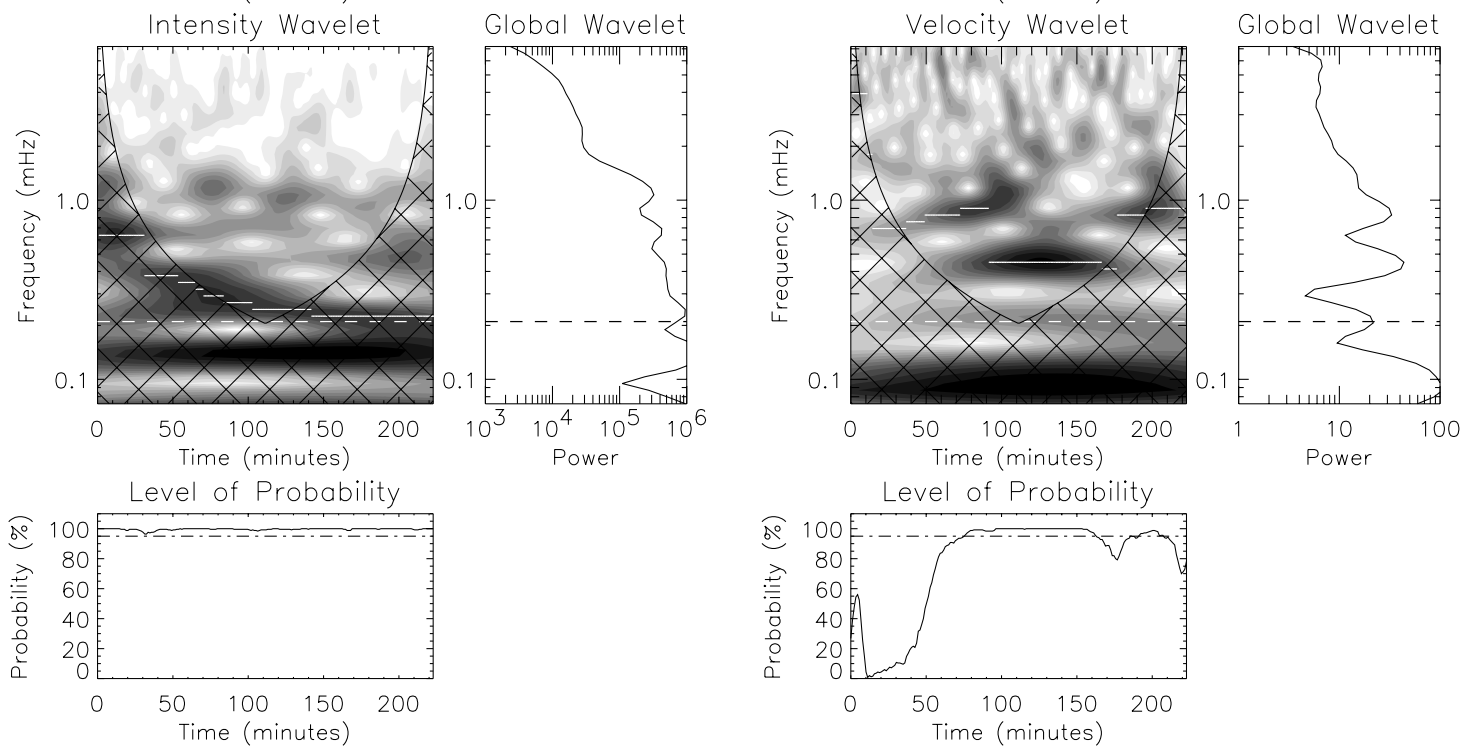

Fig. 4. The results of the wavelet analysis corresponding to the O IV $554 \AA$ line at pixel 40 in s18831r00. 


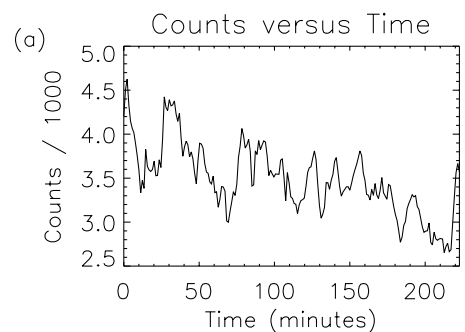

Global freq. ot max. power $(>0.21$
$=0.25 \mathrm{mHz}$ Prob. level: $99-100 \%$
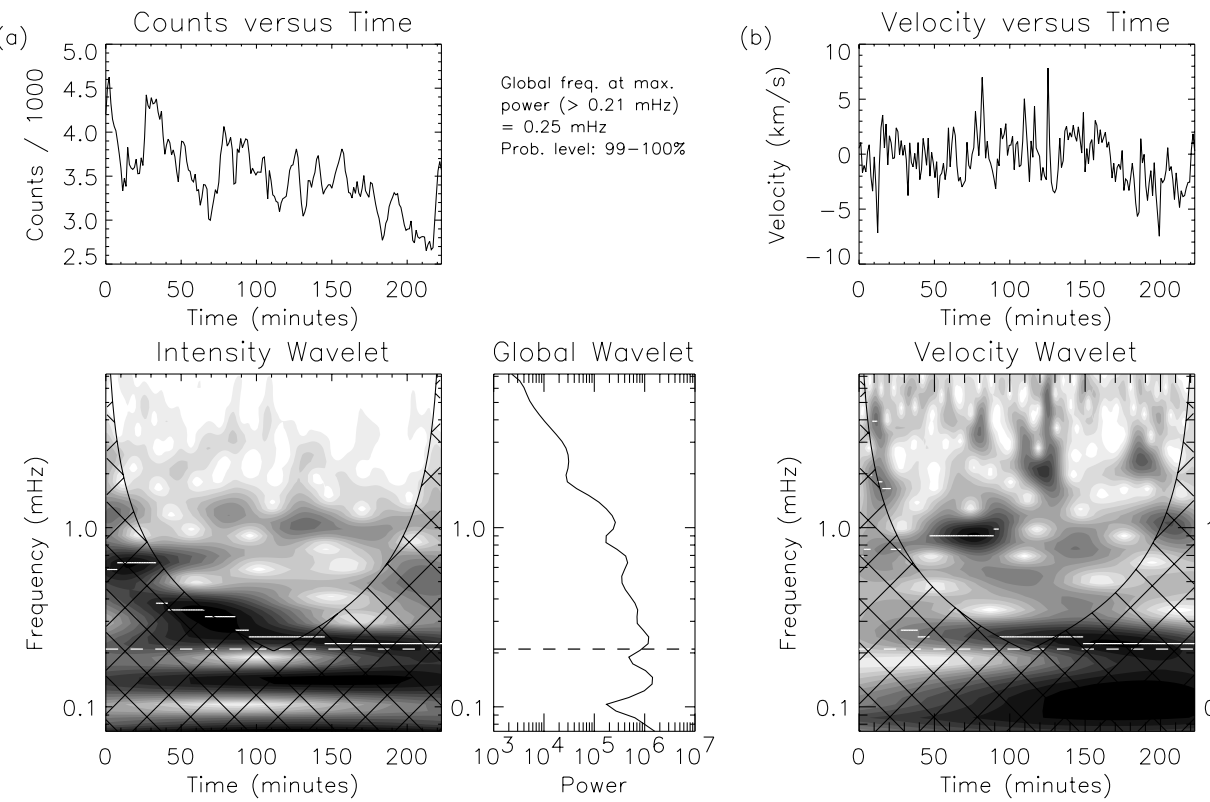

Global freq. at max. power $(>0.21 \mathrm{~m}$
$=0.25 \mathrm{mHz}$

Prob. level: $99-100 \%$
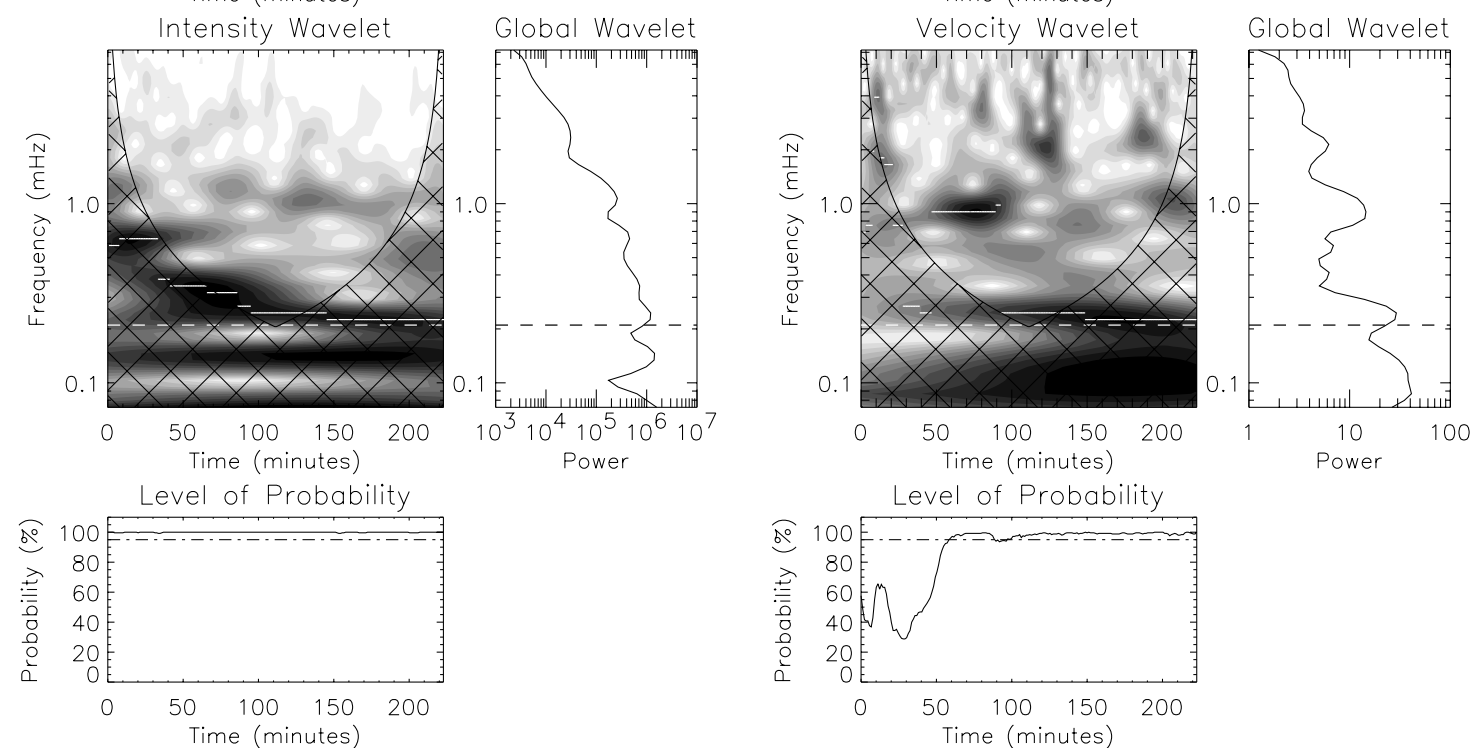

Fig. 5. The results of the wavelet analysis corresponding to the O v $629 \AA$ A line at pixel 40 in s18831r00.

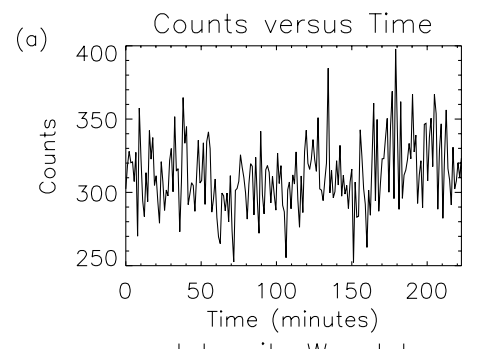

Global freq. at $\max$.
power $(>0.21 \mathrm{mHz})$ $=0.38 \mathrm{mHz}$

Prob. level: $96.8 \%$

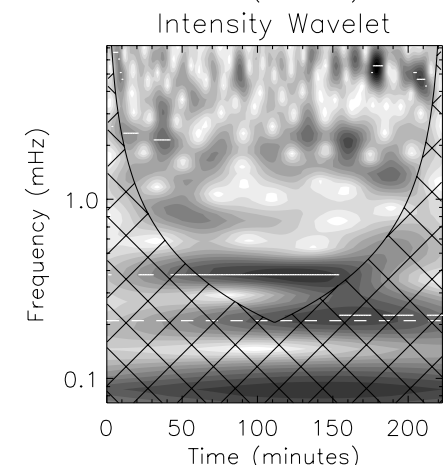

Global Wavelet
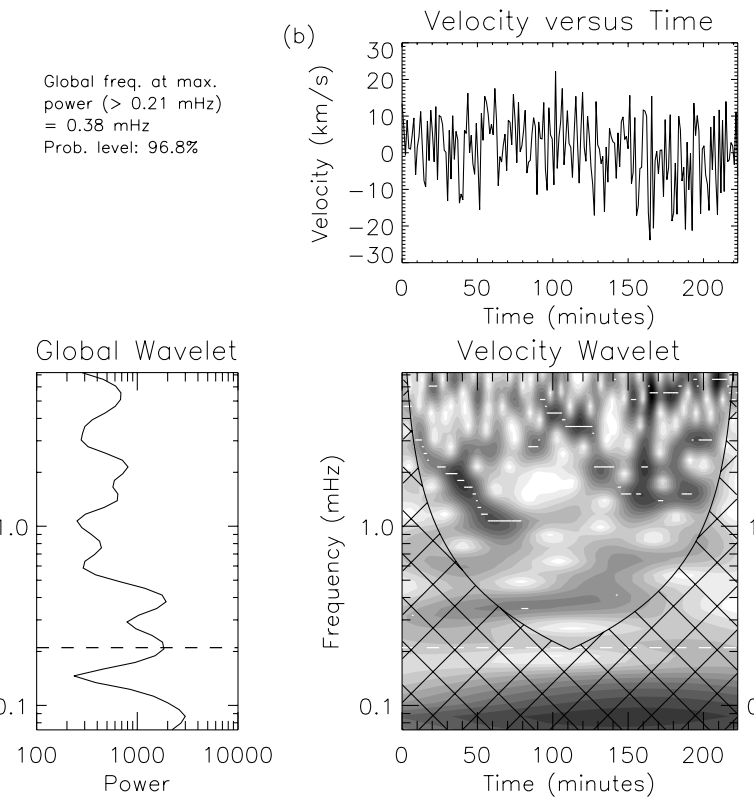

Global freq. at max. power (>0.21 mHz)

Prob. level: $0.0 \%$

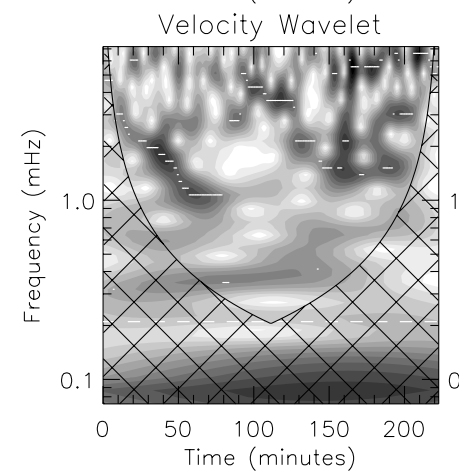

Global Wavelet
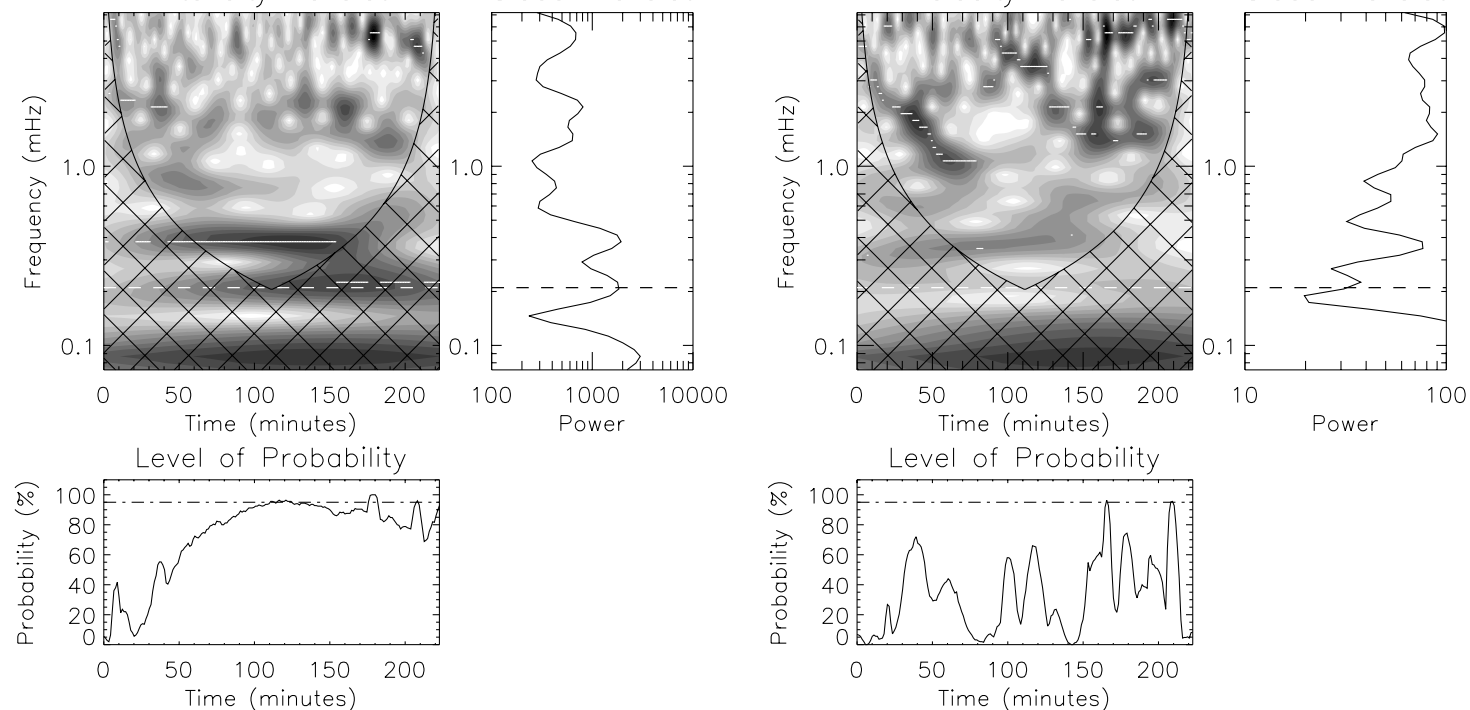

Fig. 6. The results of the wavelet analysis corresponding to the Mg Ix $368 \AA$ line and summed pixels $39-40$ in s18831r00. 
Table 1. Details of the temporal sequence CHROM_N6.

\begin{tabular}{llllll}
\hline Date & Dataset & $\begin{array}{l}\text { Pointing } \\
X, Y\end{array}$ & $\begin{array}{l}\text { Start } \\
\text { UT }\end{array}$ & $\begin{array}{l}\text { End } \\
\text { UT }\end{array}$ & Lines Observed \\
\hline & & & & & \\
14 March 2000 & s18831r00 & $92,-1051$ & $12: 06$ & $15: 50$ & He I, O III, O IV, O v, Mg IX \\
15 March 2000 & s18854r00 & $27,-1056$ & $12: 09$ & $15: 53$ & He I, O III, O IV, O v, Mg IX \\
\hline
\end{tabular}

locations of the maximum wavelet power at each particular time. The lowest panel shows the variation of the probability level over the observing time, by which it is possible to see whether the maximum wavelet power at any time in the wavelet spectrum has a high or low probability of being due to noise. Only locations that have a probability greater than $95 \%$ are regarded as being real, i.e. not due to noise. Cross-hatched regions, on either side of the wavelet spectrum, indicate the "cone of influence" (COI), where edge effects become important (see Torrence \& Compo 1998). The dashed horizontal lines in the wavelet spectra indicates the lower frequency cut-off, in this instance $0.21 \mathrm{mHz}$ (due to edge effects), corresponding to oscillations with periods of $\sim 80 \mathrm{~min}$.

The results from Fig. 2 show that the He I $584 \AA$ intensity oscillations are stronger and slightly more reliable than the velocity oscillations. The time frequency phase plane plots of intensity (left panels of Fig. 2) shows significant power around $0.3 \mathrm{mHz}$ for almost the entire observing period. There is secondary power concentrated around $0.6 \mathrm{mHz}$ for part of the sequence. The global wavelet spectrum (on the right of Fig. 2a), which is the average of the wavelet power spectrum over the entire observing period shows the strongest intensity power at $0.27 \mathrm{mHz}$. This is printed out in Fig. 2 above the global wavelet plot, together with the probability estimate for the global wavelet power spectrum. The velocity curve rarely shows power above the $90 \%$ level and thus can not be considered as being signifcant.

Now we turn our attention to several oxygen lines formed at transition region temperatures. The wavelet results of the O III $599 \AA$, O IV $554 \AA$ and O V $629 \AA$ lines are presented in Figs. 3, 4 and 5, respectively. The O III $599 \AA$ line shows intensity power in the $0.25-1.2 \mathrm{mHz}$ range with a peak at $0.25 \mathrm{mHz}$ in the global wavelet spectrum at a high probability level (see value in figure and in Table 2). The velocity oscillation shows a similar trend with a varying probability level. The strongest global peak is at $0.41 \mathrm{mHz}$, with a high probability level. The O IV line (see Fig. 4) shows strong intensity power in the $0.2-$ $1.2 \mathrm{mHz}$ range with the strongest power peaks at $0.25 \mathrm{mHz}$ and $0.41 \mathrm{mHz}$. The velocity peaks at 0.45 and $0.83 \mathrm{mHz}$ both with a high probability level. The O v $629 \AA$ intensity shows a similar power distribution with the strongest power peak at $0.25 \mathrm{mHz}$, while the velocity shows the strongest powers at 0.25 and $0.9 \mathrm{mHz}$. Both are present with a very high probability level (see Table 2). Note that the nature and period of oscillations, corresponding to the three oxygen lines formed over a range of transition region temperatures, behaves more or less in a similar way.

Next we present the wavelet results for the coronal line Mg IX $368 \AA$ in Fig. 6. Because of the low signal to noise ratio in this case we binned over two pixels (39-40). The global wavelet spectrum shows that the strongest intensity power peak is around $0.38 \mathrm{mHz}$, with a high probability level. The velocity signal for this line is too weak for any reliable detection of oscillations.

Now, to emphasise the fact that these low frequency oscillations are not only from this particular pixel location but rather from all over the inter-plumes across our slit, and also from the disk part of the coronal hole, we show the spatial behaviour of the oscillation frequencies measured from the He I $584 \AA$ \& O v $629 \AA$ lines in Figs. 7 and 8 , respectively. These figures show the measured frequencies as a function of position along the slit (X-F slice). The frequencies in the left panels (crosses) correspond to the maximum power, which have a probability of more than the $95 \%$ after the randomisation test. The total number of counts in a pixel (summed counts) during the observation is shown in the right column, and is useful in identifying the limb (pixel 41 corresponds to the limb). It also shows the intensity fall off as we go outside the limb (pixel 40 and lower). The intensity and velocity results both show that the primary maxima in the global wavelet spectra lies in the range $0.23-0.8 \mathrm{mHz}$. The appearance of more crosses in the intensity $\mathrm{X}-\mathrm{F}$ slices as compared to the velocity also indicates that the intensity oscillations are stronger and more reliable ( $>95 \%$ probability level). It might also indicate that the intensity oscillations are more coherent than the velocity, but it should be borne in mind that the velocity oscillations are weak, so it might be that they are there, but the randomisation test does not allow them to be detected over $95 \%$ probability level. Furthermore it is interesting to note that these low frequency oscillations are also present on the disk part (very close to the limb, e.g. px 44) just below the inter-plume lane. This gives an indication that whatever is causing these oscillations in the inter-plumes, is also present on the bright part of the disk, presumably in the network boundaries.

As an alternative method of representing the presence of these long period oscillations we show in Fig. 9, the intensity variation of $\mathrm{He}$ I $584 \AA$ line at different locations (off-limb) in the inter-plumes. Just from a visual 


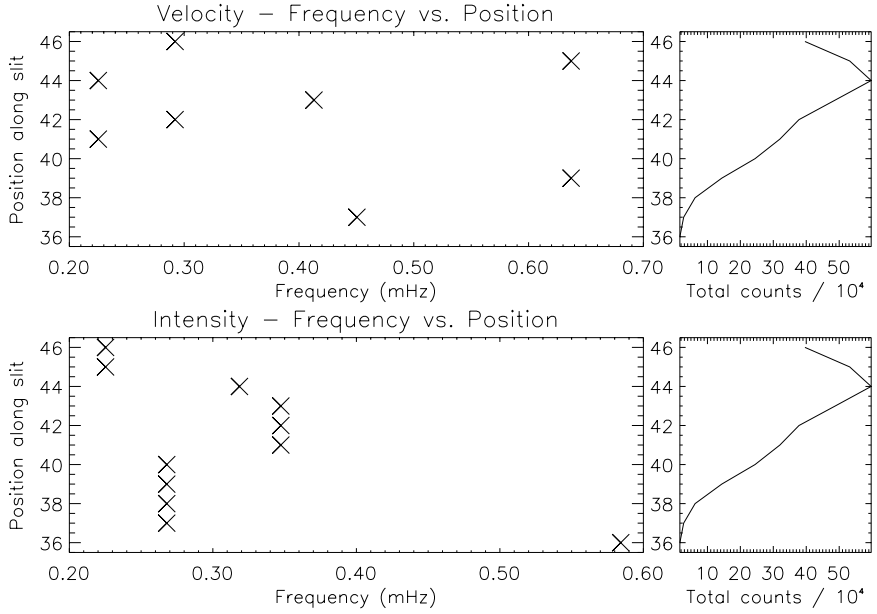

Fig. 7. Frequencies measured in the intensity (bottom row) and velocity (top row) fluctuations of the He I $584 \AA$ line, as a function of spatial position along the slit. The left panels show the frequencies corresponding to the maximum power measured above the $95 \%$ confidence level, after the randomisation test, in the global wavelet plot. The right panels show the total number of counts in a pixel (summed counts) over the observation time.
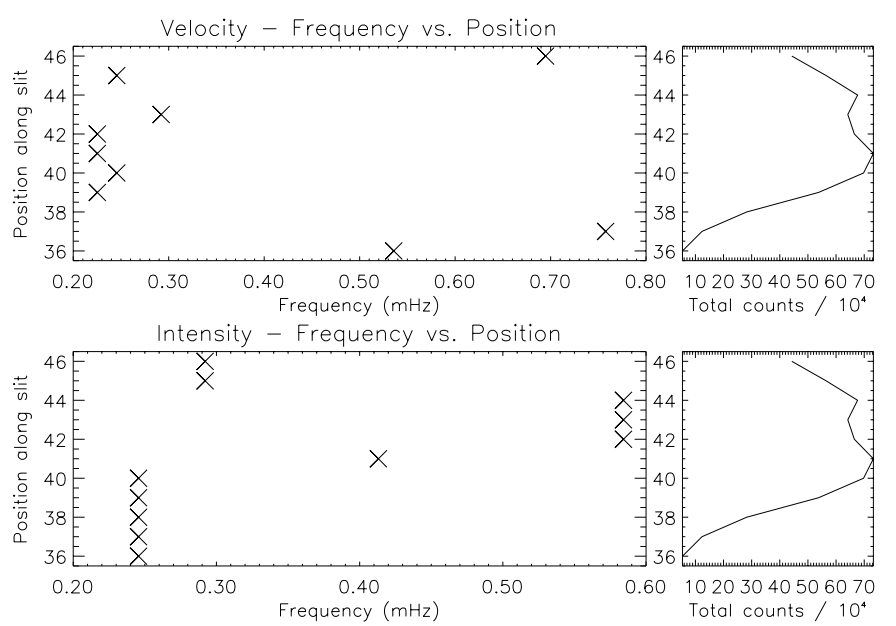

Fig. 8. Frequencies in the O v $629 \AA$ line, as a function of spatial position along the slit.

examination of the He I $584 \AA$ light-curves (Fig. 9 and top panel of Fig. 2) one can see the presence of a long periodicity of $\sim 70 \mathrm{~min}$ (notice the three strong peaks), which becomes more apparent further off-limb, up to at least 25 arcsec.

Now we turn our attention to dataset s18854r00. We first show the space time behaviour (a portion of the slit which focuses the inter-plume region and the coronal hole) as observed by He I $584 \AA$, in the form of a X-T slice (Fig. 10). The top panel shows the original intensity map. To bring out the details of the original intensity map (X-T slice) we have filtered out the bright components in the image. The intensity map $I(y, t)$ is convolved in the time direction with a Gaussian $G(t)$. This results in a smoothed image $S(y, t)=I * G$ which contains no high frequencies. Then dividing the original intensity map by the
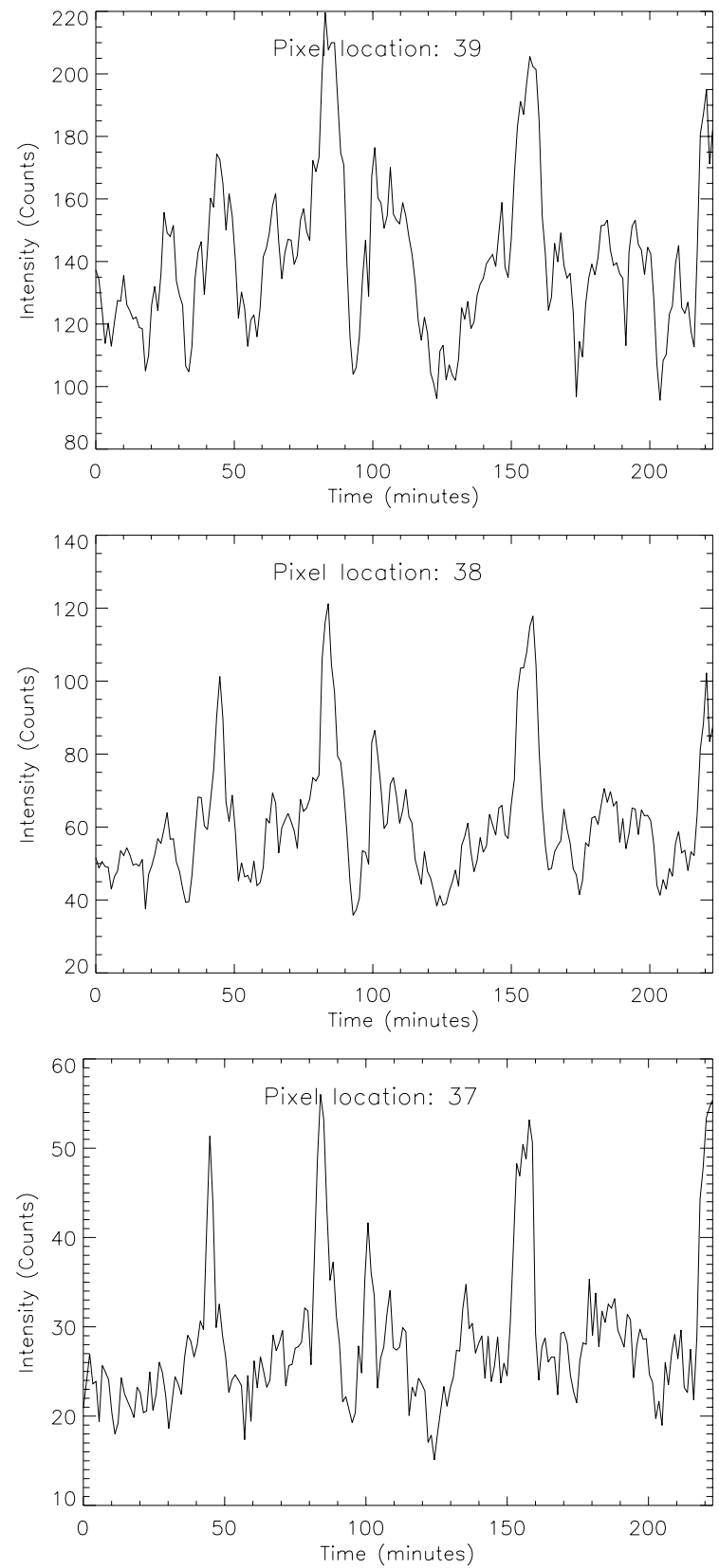

Fig. 9. Intensity variation of He I $584 \AA$ line in the s18831r00 dataset at different inter-plume locations (off-limb) as labelled.

smoothed map results in the contrast enhanced map, i.e. $C(y, t)=I(y, t) / S(y, t)$ (see Doyle et al. 1999, for details). In this contrast enhanced image (lower panel of Fig. 10), the solar north-south (SOLAR_Y) direction is in the vertical axis, the horizontal axis is time. Once again the right panels show the total number of counts in a pixel. The grey scale coding has the most intense regions as white. Note that around the time 20th, 70th and 110th min of the observing sequence there are very bright features detected in the $\mathrm{X}-\mathrm{T}$ slice, perhaps spicules? The gradients of these structures (in X-T slice) can provide information about their speeds. Banerjee et al. (2000b) have however studied their structures and dynamics in greater detail so we do not intend to go any further in that direction here. 


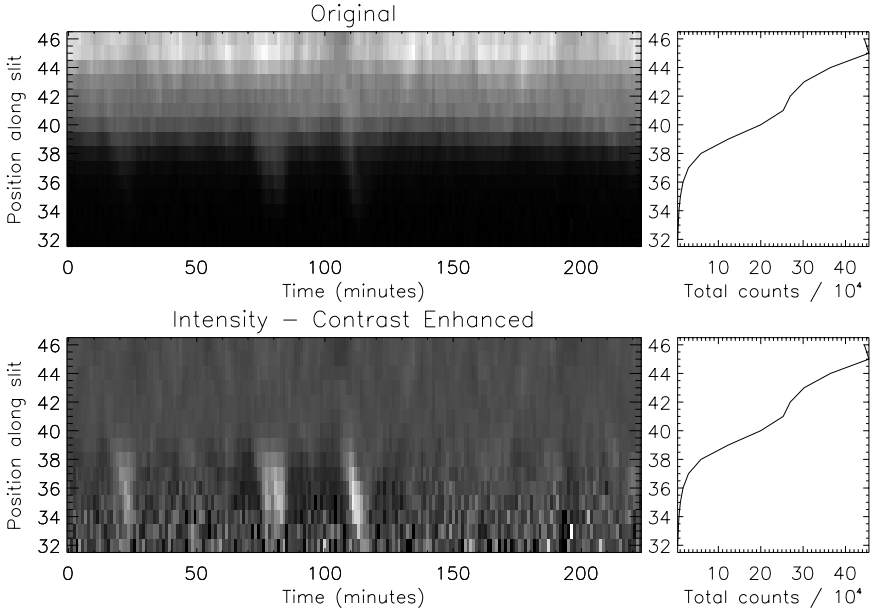

Fig. 10. Space time behaviour of the intensity in the He I $584 \AA$ line corresponding to the s18854r00 dataset. The left panels show the intensity maps (X-T slice) and the right panels show the summed counts. The gray scale coding has the most intense regions as white.

The presence of these jet-like features strongly affects the background oscillations and thus our main objective of studying the oscillations of the inter-plume regions was not possible for this dataset. However, the disk part of the slit has not been affected by the presence of these features and so we use it here to investigate oscillations on the disk. For a representative case of the coronal hole oscillations, corresponding to a network bright pixel we choose px 45 of s 18854 r00 dataset. For He I $584 \AA$, the intensity and velocity both show the strongest peak at $0.23 \mathrm{mHz}$. For O v $629 \AA$ the intensity and velocity both shows strong peaks around 0.27 and $0.25 \mathrm{mHz}$ respectively, all with a high probability level and also lasting for almost the entire observing period. The detailed results from the other lines and also from brightest pixel on the disk (px 44) for the dataset s18831r00, are summarised in Table 3. It is clear that the long period oscillations are present in all the lines and in both datasets.

\section{Discussion}

Recent UVCS/SoHO observations (Giordano et al. 2000; Patsourakos \& Vial 2000) have established that the interplume lanes provide a channel for the acceleration of the fast solar wind. Patsourakos \& Vial (2000) have reported a total outflow velocity of $\sim 67 \mathrm{~km} \mathrm{~s}^{-1}$ at $1.05 R_{\odot}$, as measured by SUMER/SoHO. Their result is consistent with large oxygen ion outflow velocities of 105 and $150 \mathrm{~km} \mathrm{~s}^{-1}$ at $1.7 R_{\odot}$ as measured by UVCS/SoHO (Giordano et al. 2000). They have also shown that in the inter-plume lanes the outflow is much faster than in the plumes, where the outflow velocity is between 0 and $65 \mathrm{kms}^{-1}$. Thus it is becoming important to know whether one can find different type of waves in these two regions, namely the plume and the inter-plume. Furthermore since inter-plume and network seems to be the main source for the fast solar wind when viewing off-limb and on disk respectively, it is also important to study them individually and finally to find a connection between these two regions. Highcadence EIT/SoHO observations indicate that quasi periodic fluctuations with periods of 10-15 min are present in polar plumes (DeForest \& Gurman 1998) with a filamentary structure within the plume, on a spatial scale of 3-5 arcsec. These authors conclude that the waves are either sound waves or slow magneto-acoustic waves, propagating along the plumes at $\sim 75-150 \mathrm{kms}^{-1}$. Recently, Ofman et al. (2000a) detected quasi periodic variations in the polarization brightness $(\mathrm{pB})$ at $1.9 R_{\odot}$, in both plume and inter-plume regions. Their Fourier power spectrum shows significant peaks around $1.6-2.5 \mathrm{mHz}$ and additional smaller peaks at longer and shorter time-scales. Their wavelet analysis of the $\mathrm{pB}$ time series shows that the coherence time of the fluctuations is about $30 \mathrm{~min}$.

Compressional modes reveal themselves in the form of intensity oscillations, through variations in the emission measures, and because of density fluctuations they are detected as velocity oscillations as well. This fact allows us to interpret the oscillations measured here as being due to slow magneto-acoustic waves. We should also point out that the wavelet analysis suffers from edge effects at both ends of the time series. Some of our low frequency oscillations in the phase plots are very close to the COI, but at the same time the primary and secondary peaks in the global wavelet spectra are above the lower frequency cut off of $0.21 \mathrm{mHz}$. So our detected oscillation frequencies above $95 \%$ probability level (see Tables 2 and 3 ) are quite reliable. Also note that the velocity oscillations are not always reliable (e.g. Table 2, He I, Mg IX). It is further interesting to note that these long period slow waves are present all the way from the chromosphere, through the transition region to the corona (although the coronal $\mathrm{Mg}$ IX line is much less reliable). It is likely that the waves detected at $1.9 R_{\odot}$ by Ofman et al. $(1997,2000 \mathrm{a})$ using UVCS/SoHO and the waves detected by DeForest \& Gurman (1998) around $1.2 R_{\odot}$ using EIT/SoHO are the same as those reported here and as also observed by CDS/SoHO in the polar plumes, very close to the solar limb and in the network boundary of the coronal holes, by Banerjee et al. (2000b,c).

It is therefore interesting to note that we are detecting similar types of waves (compressional and with long periodicities) in plumes and inter-plume regions close to the limb and also in the network boundaries in the coronal hole. Ofman et al. (2000a) have also observed these long period waves in plumes and inter-plumes at $1.9 R_{\odot}$. The periodicity in the inter-plumes seems to be slightly longer than in the plumes. Linear slow magneto-acoustic waves propagate at a speed close to the sound speed in a low $\beta$ coronal plasma. The long periodicity in the interplume may be just a temperature effect on the slow waves. Plumes are cooler than the background plasma (DeForest et al. 1997). However, we should also point out that, because of line of sight effects and the proximity of the polar plumes to our slit locations, we can not rule out contributions from both regions being present. Another possibility 
Table 2. Oscillation frequencies observed in the inter-plume a few arcsec off-limb (px 40 in our binned scale corresponding to the s18831r00 dataset). By using the wavelet method and the randomisation test the frequencies corresponding to primary and secondary peaks in the global wavelet spectrum and their corresponding probability levels are listed.

\begin{tabular}{|l|llll|llll|}
\hline \multirow{2}{*}{ Lines } & \multicolumn{3}{|c|}{ Intensity Results } & \multicolumn{3}{c|}{ Velocity results } \\
\cline { 2 - 8 } & Primary & Prob. level & Secondary & Prob. level & Primary & Prob. level & Secondary & Prob.level \\
\hline He I & $0.27 \mathrm{mHz}$ & $99-100 \%$ & $0.58 \mathrm{mHz}$ & $99-100 \%$ & & & & \\
O III & $0.25 \mathrm{mHz}$ & $99-100 \%$ & $0.64 \mathrm{mHz}$ & $99-100 \%$ & $0.41 \mathrm{mHz}$ & $98.0 \%$ & & \\
O IV & $0.25 \mathrm{mHz}$ & $99-100 \%$ & $0.41 \mathrm{mHz}$ & $99-100 \%$ & $0.45 \mathrm{mHz}$ & $99-100 \%$ & $0.83 \mathrm{mHz}$ & $99-100 \%$ \\
O v & $0.25 \mathrm{mHz}$ & $99-100 \%$ & $0.35 \mathrm{mHz}$ & $99-100 \%$ & $0.25 \mathrm{mHz}$ & $99-100 \%$ & $0.9 \mathrm{mHz}$ & $99.2 \%$ \\
Mg IX & $0.38 \mathrm{mHz}$ & $96.8 \%$ & & & & & & \\
\hline
\end{tabular}

Table 3. Summary of the oscillation frequencies observed corresponding to the brightest pixel on-disk (px 44 in our binned scale for s18831r00 dataset and px 45 of s18854r00 dataset).

\begin{tabular}{|c|c|c|c|c|c|c|c|c|c|}
\hline \multirow[t]{2}{*}{ Dataset } & \multirow[t]{2}{*}{ Lines } & \multicolumn{4}{|c|}{ Intensity Results } & \multicolumn{4}{|c|}{ Velocity results } \\
\hline & & Primary & Prob. level & Secondary & Prob. level & Primary & Prob. level & Secondary & Prob. level \\
\hline \multirow[t]{4}{*}{ s18854r00 } & He I & $0.23 \mathrm{mHz}$ & $99-100 \%$ & $0.38 \mathrm{mHz}$ & $99-100 \%$ & $0.23 \mathrm{mHz}$ & $95.6 \%$ & $0.58 \mathrm{mHz}$ & $99-100 \%$ \\
\hline & O III & $0.25 \mathrm{mHz}$ & $99-100 \%$ & $0.64 \mathrm{mHz}$ & $99-100 \%$ & $0.29 \mathrm{mHz}$ & $98.8 \%$ & $0.83 \mathrm{mHz}$ & $99.6 \%$ \\
\hline & $\mathrm{O} \mathrm{IV}$ & $0.27 \mathrm{mHz}$ & $99-100 \%$ & $0.98 \mathrm{mHz}$ & $99-100 \%$ & $0.32 \mathrm{mHz}$ & $99-100 \%$ & $0.76 \mathrm{mHz}$ & $99-100 \%$ \\
\hline & $\mathrm{O} v$ & $0.27 \mathrm{mHz}$ & $99-100 \%$ & $0.58 \mathrm{mHz}$ & $99-100 \%$ & $0.25 \mathrm{mHz}$ & $99-100 \%$ & $0.58 \mathrm{mHz}$ & $99-100 \%$ \\
\hline \multirow[t]{5}{*}{ s18831r00 } & He I & $0.32 \mathrm{mHz}$ & $99-100 \%$ & $0.9 \mathrm{mHz}$ & $99-100 \%$ & $0.23 \mathrm{mHz}$ & $99-100 \%$ & & \\
\hline & $\mathrm{O}$ III & $0.58 \mathrm{mHz}$ & $97.2 \%$ & & & & & $0.98 \mathrm{mHz}$ & $99.2 \%$ \\
\hline & O IV & $0.58 \mathrm{mHz}$ & $95.6 \%$ & $0.29 \mathrm{mHz}$ & $99-100 \%$ & $0.23 \mathrm{mHz}$ & $98.0 \%$ & $0.29 \mathrm{mHz}$ & $98.0 \%$ \\
\hline & $\mathrm{O} v$ & $0.58 \mathrm{mHz}$ & $97.2 \%$ & $0.29 \mathrm{mHz}$ & $99-100 \%$ & $0.45 \mathrm{mHz}$ & $99-100 \%$ & & \\
\hline & Mg IX & $0.41 \mathrm{mHz}$ & $96.8 \%$ & & & & & & \\
\hline
\end{tabular}

is that the waves detected adjacent to a plume structure could simply be waves which just leaked out of the plume cavity and are propagating through the inter-plume.

Patsourakos \& Vial (2000) concluded that a solar wind outflow starting from the network and then continuing in the inter-plume is a reasonable conceptual description. Our observation of a long periodicity in the network and in the inter-plumes (as presented here) supports this description. The likely detection of compressional waves in the south polar coronal hole close to the limb (as presented here) and well above the limb (Ofman et al. 2000a) may provide the additional momentum and heat required for the acceleration of the fast solar wind in coronal holes. From our observation it is clear that the velocity amplitudes are quite small and they appear to be sub-sonic, thus the linear theory may well be applicable (at least close to the limb). The energy flux carried by the slow magneto-acoustic waves can be estimated as $\rho\left[(\delta v)^{2} / 2\right] v_{\mathrm{s}}$, in WKB approximation, where $\delta v$ is the wave velocity amplitude, and $c_{\mathrm{S}}$ is the sound speed. Using $c_{\mathrm{S}}=150 \mathrm{~km} \mathrm{~s}^{-1}$ in the low $\beta$ coronal plasma, $\rho=1.67 \times 10^{-15} \mathrm{gm} \mathrm{cm}^{-3}$, and $\delta v=10 \mathrm{kms}^{-1}$, we get a wave energy flux of $\sim 1.25 \times 10^{4} \mathrm{ergs}_{\mathrm{cm}}^{-2} \mathrm{~s}^{-1}$. This is almost an order of magnitude less than $\sim 10^{5} \mathrm{ergs}^{-2}$, the energy flux required to accelerate the fast solar wind. These waves can be further damped by compressive viscosity (Ofman et al. 2000b).

Finally our possible detection of long period slow waves in plume and inter-plume regions raises further questions on the magnetic topology of these regions and the influence of magnetic field. Most of the theoretical progress so far has been devoted to waves in plumes (Ofman et al. 2000b; Nakariakov et al. 2000), where the linear and nonlinear effects have been included for the study of the wave propagation. Such numerical simulations for inter-plumes are very much warranted.

Acknowledgements. We would like to thank the referee for his valuable suggestions. Research at Armagh Observatory is grant-aided by the N. Ireland Dept. of Culture, Arts and Leisure. D. B. wishes to thank the ONDERZOEKSRAAD of K.U. Leuven for a fellowship $(\mathrm{F} / 99 / 42)$ and $\mathrm{EOS}$ is a member of the European Solar Magnetometry Network

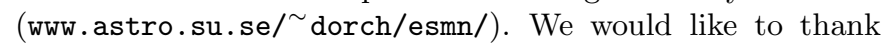
the CDS and EIT teams at Goddard Space Flight Center for their help in obtaining the present data. CDS and EIT are part of SOHO, the Solar and Heliospheric Observatory, which is a mission of international cooperation between ESA and NASA. This work was supported by PPARC grant PPA/G/S/1999/00055. The original wavelet software was provided by C. Torrence and G. Compo, and is available at URL: http://paos. colorado.edu/research/wavelets/.

\section{References}

Ahmed, I. A., \& Withbroe, G. L. 1977, Sol. Phys., 53, 397

Banerjee, D., O'Shea, E., Doyle, J. G., \& Goossens, M. 2001, A\&A, 371, 1137

Banerjee, D., Teriaca, L., Doyle, J. G., \& Lemaire, P. 2000a, Sol. Phys., 194, 43 
Banerjee, D., O’Shea, E., \& Doyle, J. G. 2000b, A\&A, 355, Ofman, L., Romali, M., Poletto, G., Noci, G., \& Kohl, J. L. 1152 1997, ApJ, 491, L111

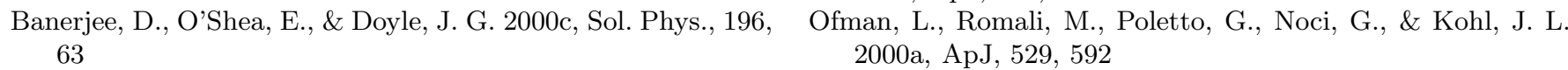

DeForest, C. E., Plunkett, S. P., \& Andrews, M. D. 2001, ApJ, Ofman, L., Nakariakov, V. M., \& Selegals, N. 2000b, ApJ, 533, 546, 569 1071

DeForest, C. E., Hoeksema, J. T., Gurman, J. B., et al. 1997, Sol. Phys., 175, 393

DeForest, C. E., \& Gurman, J. B. 1998, ApJ, 501, L217

Doyle, J. G., van den Oord, G. H. J., O’Shea, E., \& Banerjee, D. 1999, A\&A, 347, 335

Giordano, S., Antonucci, E., Noci, G., Romali, M., \& Kohl, J. L. 2000, ApJ, 531, L79

Harrison, et al. 1995, Sol. Phys., 162, 233

Hassler, D. M., Wilhelm, K., Lemaire, P., \& Schühle, U. 1997, Sol. Phys., 175, 375

Koutchmy, S., \& Bocchialini, K. 1998, Solar Jets \& Polar plumes ESA, SP-421, 51

O'Shea, E., Banerjee, D., Doyle, J. G., Fleck, B., \& Murtagh, F. 2001, A\&A, 368, 1095

Patsourakos, S., \& Vial, J.-C. 2000, A\&A, 359, L1

Saito, K. 1965, PASJ, 17, 1

Torrence, C., \& Compo, G. P. 1998, Bull. Amer. Meteor. Soc., 79, 61

Wang, Y.-M. 1998, ApJ, 501, L145

Wang, Y.-M., Sheeley, N. R., \& Dere, K. D., et al. 1997, ApJ, 484, L75

Wang, Y.-M., \& Sheeley, N. R. 1995, ApJ, 452, 457

Wilhelm, K., Marsch, E., Dwivedi, B. N., et al. 1998, ApJ, 500, 1023

Nakariakov, V. M., Ofman, L., \& Arber, T. D. 2000, A\&A, Young, P. R., Klimchuk, J. A., \& Mason, H. E. 1999, A\&A, 353,741

Nemec, A. F., \& Nemec, J. M. 1985, AJ, 90, 2317 350, 286 